Case report

Open Access

\title{
Hip screw lateral migration with no cut-out or non-union implication: a case report
} Nikolaos Lasanianos ${ }^{1 *}$, Georgios Mouzopoulos ${ }^{2}$ and Ioannis Georgilas ${ }^{3}$

\author{
Addresses: ${ }^{1} 1^{\text {st }}$ Trauma and Orthopaedic surgery department "Evangelismos" General Hospital of Athens Lontou 12, Palea Penteli, 15236, \\ Athens-Greece, ${ }^{2} 1^{\text {st }}$ Trauma and Orthopaedic surgery department, "Evangelismos" General Hospital of Athens, Sofokli Venizelou 23, \\ Peristeri 12131, Athens-Greece and ${ }^{3} 1^{\text {st }}$ Trauma and Orthopaedic surgery department, "Evangelismos" General Hospital of Athens, Ypsilantou 45, \\ 10676, Athens-Greece \\ Email: NL* - lasagrc@hotmail.com; GM - gmouzopoulos@yahoo.gr; IG - giannisgeor@yahoo.gr \\ * Corresponding author
}

Published: 10 March 2009

Cases Journal 2009, 2:6419 doi: 10.1186/1757-1626-2-6419
Received: 6 January 2009

Accepted: 4 February 2009

This article is available from: http://casesjournal.com/casesjournal/article/view/2/3/6419

(C) 2009 Lasanianos et al; licensee Cases Network Ltd.

This is an Open Access article distributed under the terms of the Creative Commons Attribution License (http://creativecommons.org/licenses/by/3.0), which permits unrestricted use, distribution, and reproduction in any medium, provided the original work is properly cited.

\begin{abstract}
Hip screw migration of peritrochanteric fracture fixation devices is a described complication in English literature. Medial migration occupies the majority of these cases whereas lateral migration is rare. We report the case of an 85-year-old woman whose intramedullary osteosynthesis of a trochanteric fracture was complicated by hip screw lateral migration. Mobilization was not influenced and no cut-out or non-union was detected. The migrated hip screw was easily removed and the discomfort vanished. The need for adequate surgical technique and radiographic examination after reinjuries even if the patient remains ambulatory is emphasised.
\end{abstract}

\section{Introduction}

Fractures of the trochanteric region of the femur are very common in the elderly. Many fixation devices have been developed for these fractures; the most widely used being the numerous versions of intramedullary nails which tend to replace the sliding nail plate systems. Intra-operative blood loss and operating time is much minimised, immediate load-bearing is ensured, and postoperative morbidity remains low [1]. Despite the good and reliable results, some typical failures and complications may occur $[2,3]$.

One of the most common complications of intramedullary systems is lag screw migration combined with hardware cut-out or non-union $[4,5]$. Herein we present the rare case of a laterally migrated hip screw of an intramedullary device after the fixation of a peritrochanteric fracture. Up to our knowledge this is the first case of a lateral migration in a single hip screw fixation device, without an accompanying non-union or cut-out.

\section{Case presentation}

An 85-year-old woman presented with a hip fracture after a fall. The radiographic control revealed a type 31-A.2.2, secondary to the AO classification, intertrochanteric fracture (Figure 1a) of the right femur. The fracture was fixed internally with a $130^{\circ}$ angle, $180 \mathrm{~mm}$ length ATN Trochanteric nail (DePuy, Johnson \& Johnson Company). 
A $100 \mathrm{~mm}$ length and $10.5 \mathrm{~mm}$ diameter lag screw was used and distal dynamic locking was performed. Reaming was performed to the femoral head before the placement of the lag screw and to the medullary canal before the insertion of the nail. Although the ATN system provides an optional anti-rotation screw (placed above the lag screw into the femoral head) this was not used. Postoperative $\mathrm{x}$ rays showed very good fracture reduction. The Garden alignment index was $160^{\circ}$ in the anteroposterior (AP) view (Figure 1b) and $180^{\circ}$ in the lateral view (Figure1c) which consist the absolute desirable values. The lag screw position was ideal as seen in the post-op AP and lateral view. The Tip Apex Distance (TAD) was considered to be no more than $10 \mathrm{~mm}$. Partial weight bearing was advised for 2 weeks postoperatively and then full weight bearing was allowed. The patient's mobilization program was developing normally without pain restriction or other walking difficulties. Scheduled radiographic control performed 2.5 months post surgery (Figure $2 \mathrm{a} \& 2 \mathrm{~b}$ ) revealed a slight lag screw backsliding of about $10 \mathrm{~mm}$. At that point full callus formation was achieved and the patient was advised not to restrain from her daily activities. Two weeks after the last examination the patient sustained a fall. A new radiographic control revealed a total lateral migration of the lag screw (Figure 3a). No radiographic signs of cutout, re-fracture or non-union were noticed. Swelling of the right hip was noticed the following days (Figure $3 \mathrm{~b}$ ) and the patient started complaining of night pain during bed time. Despite these findings she remained ambulatory and did not face any problems with her mobilization. The migrated lag screw was removed under local anaesthesia on the basis of one day surgery about one month after the new injury. During the screw removal drainage of about $300 \mathrm{ml}$ of post-traumatic haematoma and molten fats from the subcutaneous hip was performed (Figure 4). The nail was left in place and the wound was closed.
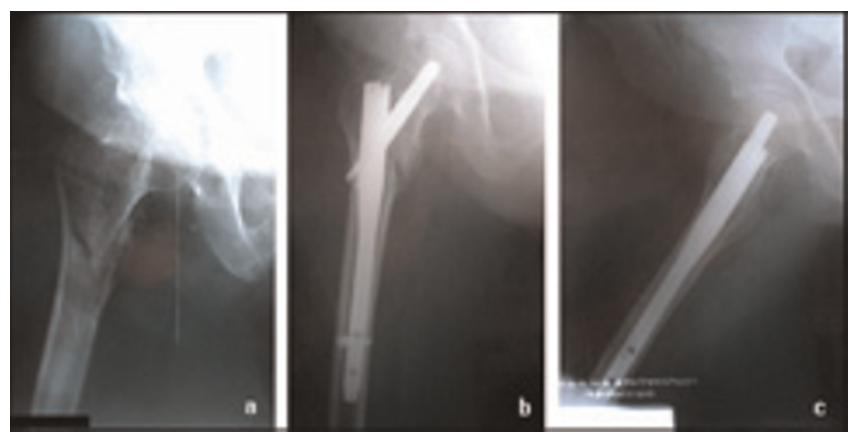

Figure I.

(a) A type 31-A.2.2 left hip intertrochanteric fracture.

(b) Postoperative anteroposterior view of the fracture shown on figure Ia. (c) Postoperative lateral view of the fracture shown on figure la.
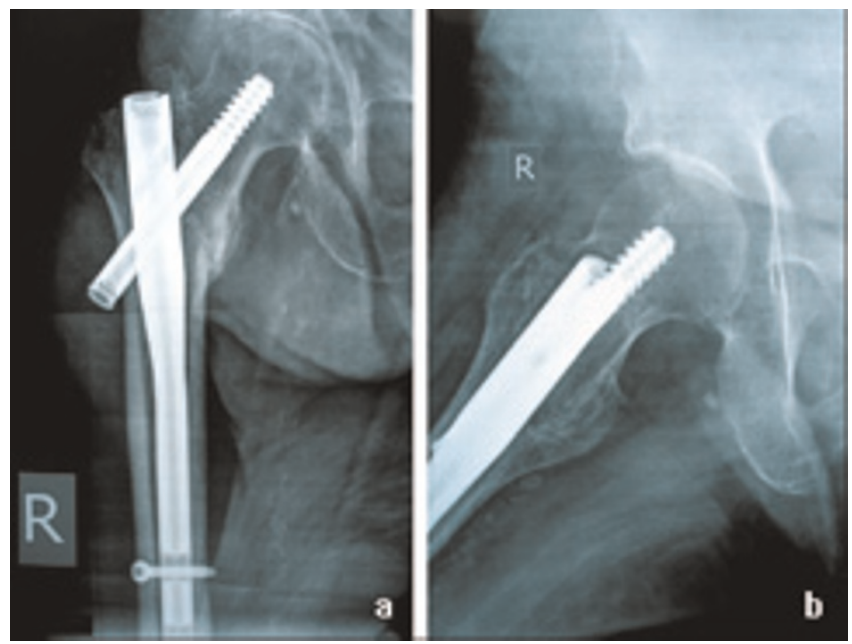

Figure 2.

(a) 2.5 months post surgery anteroposterior view showing a slight lag screw backsliding of about $10 \mathrm{~mm}$. (b) 2.5 months post surgery lateral view showing a slight lag screw backsliding of about $10 \mathrm{~mm}$.

\section{Discussion}

Peritrochanteric fractures represent a significant risk in every age group. Moreover in the elderly they may represent a risk to life. Intramedullary nailing is widely used for fixation of such fractures ensuring less operating time, minimized wounds, immediate weight bearing, faster mobilization and thus less morbidity [1]. Nevertheless complications never cease existing.

The most common and well-documented mechanical complication of these devices are: a) cut-out of the hip screw through the femoral head with varus collapse of the fracture [6]; b) fracture of the femoral shaft distal to the tip
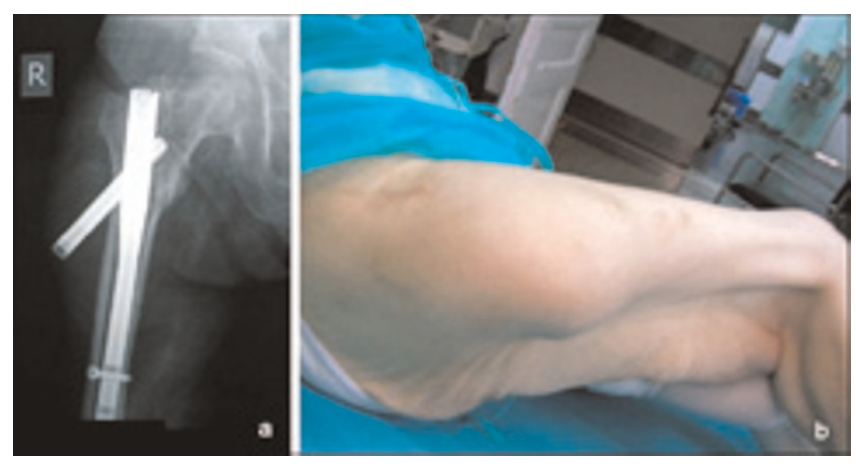

Figure 3.

(a) 3 months post surgery anteroposterior radiographic view showing full lateral migration of the hip screw after a new fall. (b) Clinical view of the swollen right hip. 


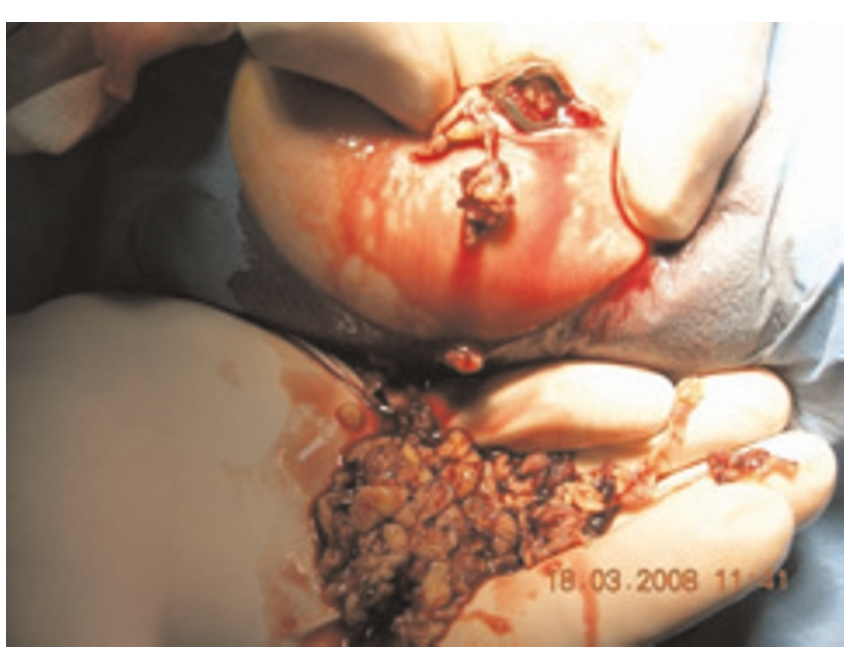

Figure 4.

Intra-operative photo of the hip screw removal showing the amount of post-traumatic haematoma and molten fats.

of the implant, a phenomenon reduced by the newer nail designs [7]; c) medial migration of the hip screw into the pelvis which is more rarely reported. The latter has been termed the "Z-effect" and has been described primarily for two-screw devices such as the proximal femoral nail (PFN, Synthes, Switzerland) [8]. Nevertheless, it has occurred in implants with a single femoral head fixation element as well, including the trochanteric fixation nail (TFN), Gamma, Zimmer, and Depuy nails [3, 9-12].

Lateral migration is more rare than medial. Up to our knowledge there are not any published cases of lateral migration, in a single use of a hip screw, without a new fracture or a cut-out accompanying the migration. Studies concerning the use of perhaps the most studied nail (Gamma nail - Howmedica) conclude that there have been no instances of implant failure without non-union or re-fracture [13].

Hip screw migration is considered to be an evolutionary complication and the ability of the implant to resist migration under dynamic loading is of critical importance. Walking subjects the implant-bone interface to combined axial and torsional loading and may play a role in lag screw migration. In our case slight, painless lateral migration (of approximately $10 \mathrm{~mm}$ ) was noticed 2.5 months post surgery and full lateral migration was noticed two weeks later after the occurrence of a new fall. The new injury caused great nail protrusion and haematoma of the hip accompanied with tenderness which gave away after the lag screw removal. We could not reach any profound explanation for the migration mechanism. The fact that the way of the lag screw into the femoral head was reamed and that the diameter of the non-threaded part of the lag screw was the same with that of the thread could provide some explanation for the behaviour of the screw and more particularly for the convenience of total migration once it was slightly detached from its original position. Moreover the osteoporotic bone of the patient did not offer an adequate grip to stabilize the sliding screw and prevent its loosening. Osteoporosis on the other hand did not seem to create any delay to bone union.

One could suppose that this is the case of a semicompleted Z-effect since the lag screw migrated laterally as if the optional anti-rotational screw (which we did not use) was there to play the role of the medially migrated half. It should be reminded here that Werner-Tutschku et al [8] were the first to describe the so called Z-effect phenomenon as the medial migration of the antirotation screw with the simultaneous lateral migration of the lag screw. The nature and aetiology of the Z-Effect phenomenon has not yet been identified. As hypothesis, a helical rotation of the antirotation screw but also an axial migration due to jerky micromotions under weightbearing are discussed. A caput-collumn-diaphysis (CCD) angle below $125^{\circ}$, osteoporosis and several attempts at reaming represent risk factors for the Z-effect phenomenon. A further explanation is the impactation of the hip pin into the proximal hole of the nail while the neck screw normally slides back during the weight-bearing period [12]. In our case it could be supposed that the non placement of the anti-rotational screw resulted in slight rotational instability of the lag screw. This may have caused the slight lateral migration that was first noticed and was completed after the mechanical impaction of the new fall.

The age of the patient, the quality of the bone, the pattern of the fracture, the stability of the reduction, the angle of the implant, and the position of the lag screw within the femoral head have all been related to this mechanism of failure, but there has been no clear consensus as to the interrelationships or relative importance of each factor. Previous clinical studies suggest that lag screws placed with a tip-apex distance (TAD) of less than $25 \mathrm{~mm}$ should rarely fail by cut-out $[14,15]$. In our case this was achieved since the TAD was measured to be less than $10 \mathrm{~mm}$. This might have been the determinant factor that resulted in early callus formation and thus relevant protection of the fractured area from lag screw micro motions and protrusion, avoiding re-fracture, non-union or excessive mechanical stress of the device.

\section{Conclusion}

Intramedullary nailing tends to become the treatment of choice for proximal femoral fractures and has a low implant failure rate. Although closed reduction and 
minimally invasive exposure minimise delayed union and non-union they are not enough for the avoidance of such complications. Caution is needed in the placement of the lag screw in manner that respects the limitations of Tip Apex Distance. A well centred lag screw contributes to better transmission of axial forces and inducts the earlier callus formation. Thus complications such the one described in this paper do not result in re-fractures, nonunion or mal-union.

\section{Abbreviations}

AP, Anteroposterior; PFN, Proximal Femoral Nail; TAD, Tip Apex Distance; TFN, Trochanteric Fixation Nail; CCD, Caput Collumn Diaphysis.

\section{Consent}

Written informed consent was obtained from the patient for publication of this case report and accompanying images. A copy of the written consent is available for review by the Editor-in-Chief of this journal.

\section{Competing interests}

The authors declare that they have no competing interests.

\section{Authors' contributions}

NL collected the data and drafted the manuscript. GM involved in revising the manuscript for important intellectual content. IG was the consultant surgeon responsible for the patient. All authors read and approved the final manuscript.

\section{References}

I. Leung, K.S., et al.: Gamma nails and dynamic hip screws for peritrochanteric fractures. A randomised prospective study in elderly patients. J Bone Joint Surg Br. 1992, 74(3):345-35I.

2. Kukla, C., et al.: The standard Gamma nail: a critical analysis of I,000 cases. J Trauma. 200I, 5 I (I):77-83.

3. Hesse, B., Gachter A: Complications following the treatment of trochanteric fractures with the gamma nail. Arch Orthop Trauma Surg. 2004, I 24(10):692-698.

4. Stocker, R., Maier R: Medial migration of lag screw in gamma nailing. Is it the result of a technical or implantation error?. Unfallchirurg. 2005, 108(I): p. 79-80

5. Tauber, M., Resch H: Sigmoid perforation after medial migration of lag screw in gamma nailing. Arch Orthop Trauma Surg. 2006, I 26(2): I |8-122.

6. Sommers, M.B. et al.: A laboratory model to evaluate cutout resistance of implants for pertrochanteric fracture fixation. J Orthop Trauma. 2004, I 8(6):36I-368.

7. Jones, H.W., Johnston P., Parker M: Are short femoral nails superior to the sliding hip screw? A meta-analysis of 24 studies involving 3,279 fractures. Int Orthop. 2006, 30(2):69-78.

8. Werner-Tutschku, W., et al.: Intra- and perioperative complications in the stabilization of per- and subtrochanteric femoral fractures by means of PFN. Unfallchirurg. 2002, I05(I0):88|-885.

9. Boldin $C$ et al.: The proximal femoral nail (PFN)-a minimal invasive treatment of unstable proximal femoral fractures: a prospective study of $\mathbf{5 5}$ patients with a follow-up of 15 months. Acta Orthop Scand. 2003, 74(I):53-58.

10. Fogagnolo, F., Kfuri M., Paccola C.A.: Intramedullary fixation of pertrochanteric hip fractures with the short AO-ASIF proximal femoral nail. Arch Orthop Trauma Surg. 2004, I 24 (I):3I-37.
II. Tyllianakis, M., et al.: Treatment of extracapsular hip fractures with the proximal femoral nail (PFN): long term results in 45 patients. Acta Orthop Belg. 2004, 70(5):444-454.

12. Papasimos, S., et al.: A randomised comparison of AMBI, TGN and PFN for treatment of unstable trochanteric fractures. Arch Orthop Trauma Surg. 2005, 125(7):462-468.

13. Yoshino, N., et al.: Implant failure of long Gamma nail in a patient with intertrochanteric-subtrochanteric fracture. J Orthop Sci. 2006, I I(6):638-643.

14. Baumgaertner, M.R., et al.: The value of the tip-apex distance in predicting failure of fixation of peritrochanteric fractures of the hip. J Bone Joint Surg Am. 1995, 77(7): I058- 064.

15. Baumgaertner, M.R., Solberg B.D.: Awareness of tip-apex distance reduces failure of fixation of trochanteric fractures of the hip. J Bone Joint Surg Br. 1997, 79(6):969-7I.

\section{Do you have a case to share?}

\section{Submit your case report today}

- Rapid peer review

- Fast publication

- PubMed indexing

- Inclusion in Cases Database

\section{Any patient, any case, can teach us something}

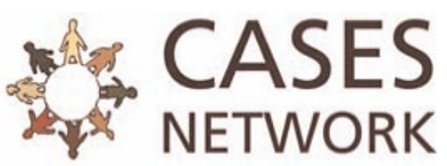

www.casesnetwork.com 